\title{
Ultrasound screening for cervical, abdominal and scrotal malignant and benign abnormalities in children
}

\section{Type}

Research paper

\section{Keywords}

ultrasound, screening, childhood cancer, No to neoplasms in children program

\begin{abstract}
Introduction

Ultrasonography plays an important role in evaluation of many diseases in pediatric population. The noninvasiveness of the method allows to its wide use in children. It is a first-line diagnostic test for detecting lymphadenopathy, benign and malignant abnormalities of the thyroid gland, abdominal structures or testes in boys. The Ultrasound Screening Program "No to Cancer in Children" was introduced to detect neoplastic lesions and possible developmental disorders in children with no symptoms to allow early diagnosis.
\end{abstract}

\section{Material and methods}

The children were scanned in special mobile ambulance - Ronald McDonald Care Mobile, equipped with two high-tech ultrasound devices. Ultrasound scans, including cervical, abdominal, pelvical and scrotal ultrasound were performed in population of asymptomatic children. In the years 2006-2019, 67.594 children, 34.892 boys and 32.702 girls aged from 9 months to 6 years were examined.

\section{Results}

Totally, 18.544 various abnormalities were detected. In case of the neck they were found in 7542 children, which represent nearly $11.2 \%$ of all patients. Changes in abdominal ultrasound were found in 4.496 cases $(6.65 \%)$. Abnormalities of the male reproductive system were detected in 6.474 boys $(18.5 \%)$. Twelve tumors were detected.

\section{Conclusions}

Screening has proved to be very useful for the early identification cancerous as well as precancerous lesions. It is also worth continuing for a reason of effective detection of other childhood anomalies. 
Ultrasound screening for cervical, abdominal and scrotal malignant and benign abnormalities in children.

Grzegorz Jedrzejewski ${ }^{1,3}$, Agnieszka Zaucha-Prazmo ${ }^{2}$, Albert Matera ${ }^{1,3}$, Magdalena Maria Wozniak ${ }^{1,3}$, Marcin Inglot $^{3}$, Mikolaj Pietkiewicz ${ }^{3}$, Andrzej Pawel Wieczorek ${ }^{1,3}$, Jerzy Kowalczyk ${ }^{2}$

${ }^{\mathbf{1}}$ Department of Pediatric Radiology, Medical University of Lublin

${ }^{2}$ Department of Pediatric Hematology, Oncology and Transplantology, Medical University of Lublin

${ }^{3}$ Top-Medical Centre, Lublin

Corresponding author:

Grzegorz Jedrzejewski

Department of Pediatric Radiology, Medical University of Lublin

Gebali 6, 20-093 Lublin, Poland

tel/fax: 48817418447

e-mail: gjedrzejewski@wp.pl

\section{Keywords}

Ultrasound; Screening; Childhood cancer; No to neoplasms in children program. 


\section{Introduction}

Ultrasonography plays an important role in evaluation of many diseases in pediatric population. The noninvasiveness of the method allows to its wide use in children. It is a first-line diagnostic test for detecting lymphadenopathy, benign and malignant abnormalities of the thyroid gland, abdominal structures or testes in boys. Also congenital anomalies, mainly of the kidney and urinary tract can be found $[1,2,3]$.

Regardless of the type of the disease, early diagnosis is essential for successful treatment, especially in case of cancer. Although cancer is not common in pediatric population, it remains one of the major causes of death in children, what was shown in studies conducted on huge pediatric cancer registries $[4,5]$. The effects of anticancer treatment depend largely on the time of diagnosis. The early detection of neoplastic lesion is essential for early diagnosis and successful treatment and allows to minimize morbidity and mortality. Although it is well known, still many tumors in pediatric population are detected in rather advanced stages (III or IV) [6]. One of the possible reason may be delayed diagnosis and thus delayed treatment $[7,8]$. However, since childhood cancer is a rare disease, the cost-effectiveness of populational ultrasound (US) screening would be low. Therefore, searching for a spectrum of abnormalities in children would be more justified.

The Ultrasound Screening Program "No to Cancer in Children" was introduced by Ronald McDonald House Charities (RMHC) Chapter in Poland in 2006 to detect neoplastic pathologies and possible developmental disorders in children with no or few symptoms to allow early diagnosis. The program was also dedicated to children from areas with poor access to general practitioners and US examinations. The introduction of the protocol was proposed by the national 
consultant in pediatric oncology and pediatric radiologists. The main goal of the program was to detect neoplastic lesions, as well as any abnormalities in the neck, abdomen pelvis and testes in boys at earlies stage, before the onset of clinical symptoms. Another goal was to reach places with difficult access to health care.

\section{Patients/Methods}

In the years 2006-2019, 67.594 children, 34.892 boys and 32.702 girls aged from 9 months to 6 years were examined for the detection of early neoplastic changes. Ultrasound examinations were performed involving the neck, the abdominal cavity, the pelvis and scrotum. Totally, 188.660 ultrasound examinations were performed, including 67.169 cervical scans, 67.448 abdominal scans, 18.822 female pelvic scans and 34.648 scrotal scans. Parents of children at particular age were invited to participate in the study voluntarily. Informed consent was received from all parents. The children were scanned in special mobile ambulance - Ronald McDonald Care Mobile, equipped with two high-tech ultrasound devices with two probes: convex C 3-7 MHz and linear L 5-9 MHz. Two experienced pediatric radiologists performed examinations simultaneously, overall fifteen pediatric radiologists took part in the project. All scans were performed according to standardized protocols.

\section{Results}

Totally, 18.544 various abnormalities were detected. In case of the neck they were found in 7542 children, which represent nearly $11.2 \%$ of all patients. In 3842 cases $(5.7 \%)$ further diagnostics was required, which included medical examinations or follow up imaging studies. These were mainly suggested in the case of enlarged lymph nodes or thyroid gland lesions. Among these changes some were suspected of 
oncological origin and then specialist consultations were advised. It was decided in 1644 patients $(2.4 \%)$. According to results of these management no malignancy in the neck area was found. The occurrence frequency of the main neck abnormalities is included in Table 1.

Changes in abdominal ultrasound were found in 4.528 cases $(6.7 \%)$, with $2.332(3.2 \%)$ urinary tract abnormalities (Table 2,3). Further proceedings were required in 2.636 patients $(3,9 \%)$, including oncologic consultation in 1175 cases $(1.7 \%)$. These mainly included focal and diffuse changes in the parenchymal organs and enlarged abdominal lymph nodes. In those patients 8 malignant tumors were detected: 1 hepatoblastoma, 6 nephroblastomas and neurogenic tumor of the rib (Table 2, 3).

Abnormalities of the male reproductive system were detected in 6.474 boys (18.5\%), out of which 3.949 patients were sent for further diagnostics $(11,4 \%), 496$ (1.4\%) with oncological suspicion. These included focal testis lesions, abnormal testis echostructure, microlithiasis and hypoplasia of the testis. Four teratomas were detected and confirmed by histological examinations. However, also patients with undescended and mobile testicles are more likely to have scrotal malignancy and should be observed in scrotal ultrasound (Table 4).

Abnormalities of the female reproductive system were quite rare, found in 246 girls $(1.3 \%)$. Only few girls $(0.002 \%)$ with enlarged inguinal lymph nodes were referred for further consultations, with no malignancy on diagnosis (Table 4).

Summarizing, in 3.315 out of 67.594 children $(4,9 \%)$ oncological processes were suspected. Twelve tumors were detected, which represent $0.02 \%$ of the examined population. All tumors were confirmed in other studies, finally by histological examinations. 
Other common pathologies of the neck concerned thyroid cyst, inhomogeneous thyroid echostructure and hypoplasia of the thyroid gland (Table 1). In case of the abdominal cavity it was enlargement of the liver, spleen and mesenteric lymph nodes (Table 2). Dilation of the collecting systems of the kidneys was the most frequent urinary tract pathology. Anatomic variants like duplication of the collecting systems were also common (Table 3). In the scrotal ultrasound mobile, undescended and hydrocele testes were dominant (Table 4).

Case report. The screening ultrasound of the abdomen was performed in almost two year old girl, with no clinical symptoms. Bilateral kidney tumors were found in early stage with diameter under $30 \mathrm{~mm}$ on the right side and $40 \mathrm{~mm}$ on the left (Figure 1). The nephroblastoma was suspected and confirmed at computed tomography (CT) examination (Supplementary Figure S2). On preoperative CT study, after month of chemotherapy the tumors showed slight decrease in volume (Figure 2). Then the preserving kidneys surgery was performed. Control magnetic resonance imaging (MRI) study of the abdomen after 6 and 11 months of observation showed only postoperative changes in both kidneys, with no recurrence (Figure 3). The standard observation is continued.

\section{Discussion}

Epidemiological studies showed higher incidence of cancers like lymphomas, central nervous system tumors and solid tumors in children and adolescents during last decades in Europe [4-9]. According to studies by Steliarova-Foucher et al., the incidence increase by about $1 \%$ per year for all cancer types [7,9]. Although epidemiological studies have not identified clear risk factors for childhood cancer so far, early diagnosis is essential to improve treatment results for childhood cancer 
[10]. Also lack of specific manifestations in the early stage of many tumors usually delays its diagnosis and prevents treatment in the early stage [11]. US screening examinations have been tried several times in the diagnosis of children, mainly in the assessment of the urinary tract $[12,13]$. Despite promising results that showed changes in approximately $25 \%$ of the examined children, these studies have not been introduced to the entire population. Another attempt was made by the RMHC Chapter in Poland, which purchased a special mobile ambulance equipped with two ultrasound rooms for pediatric ultrasound examinations [14]. These studies were cofinanced with local self-governments. The goal of the ultrasound screening programme was to identify the abnormalities in the early stage, without clinical symptoms, to allow implementation of further procedures in the earliest phase. US was used due to its non-invasiveness, what is especially important in pediatric patients. The population of children under 6 years of life was selected for exams. The experience of pediatricians to date shows that at this age some of the childhood cancers appear, which are usually detected at higher, symptomatic stages. In addition, children at this age are quite difficult to examine and cannot precisely report their symptoms. During the US examination, the child usually lies on his back, restless children can be held by parents. In the abdominal cavity, mainly parenchymatous organs such as the liver, pancreas, spleen, kidneys and organs containing fluid such as the gall bladder and urinary bladder are assessed. In girls with full bladder, reproductive organs can also be assessed. Within the neck the thyroid gland, salivary glands and lymph nodes and in boys additionally testicles, epididymis and spermatic cord are assessed. In most cases, due to the physiological image of organs, a small area of examination and a good, high-resolution images, it is a fast examination, lasting only a few minutes. Therefore, it can be carried out 
even for restless children. During the examination, parents are kept informed about the physiology and possible pathology of the observed structures and after the examination they receive the report, with suggestions what to do if abnormalities are found. These can be referrals for check-ups, family doctor or specialist consultations. In $5 \%$ of the examined children oncological processes were suspected, mostly in patients with focal lesions and enlarged lymph nodes. Thirteen malignant tumors were detected, which represent $0.02 \%$ of the examined population. Peak incidence during the earliest years of life features embryonic tumors like hepatoblastoma, nephroblastoma, or neuroblastoma. Out of these tumors the highest incidence rate is 9.3 for neuroblastoma, slightly lower for nephroblastoma -7.4 and lowest for hepatoblastoma -1.3 [15]. Based on the number of examined patients in this program and detected Wilms tumors, it gives incidence rate 9, which is higher than the incidence rate of Wilms tumor of the whole population. The incidence rate of hepatoblastoma (1.5) was similar to populational index, in contrast to the detection of neuroblastomas, which did not appear in the children examined in the Program. The example of a patient with bilateral Wilms tumor, described in the case report is very important. Finding these changes at an earlier stage allowed for a saving treatment and leaving both kidneys. So far, no recurrence has occurred during the observation.

Moreover, during the study, a large number of other abnormalities was found, with various degree of clinical significance (Table 1-4). Such a findings provide valuable information about the frequency of certain diseases in the whole population. In the neck examination inhomogeneous thyroid echostructure and hypoplasia of the thyroid gland were observed and may indicate potential thyroid dysfunction. The most common urinary tract pathology was related to dilation of collecting systems of the kidneys, reflecting potential risk of urinary tract infections and post inflammatory 
nephropathy. Renal congenital defects may give no symptoms for a long time. A significant number of abnormalities were related to scrotal pathologies. Undescended testes and microlithiasis can affect testis growth and are proven conditions predisposing to the cancer occurrence $[14,16]$.

In summary pediatric screening examinations enable the early identification of childhood malignancies as well as developmental abnormalities and therefore the early initiation of interventions whose effectiveness, especially in oncology is crucial and evidence based. Higher rate of Wilms tumors detected in our screening Program compared to the rate of diagnosed Wilms tumors in the whole population proves sense of abdominopelvic screening ultrasound in children. Due to the oncological orientation of the Program, all suspicions of malignancies are referred to the oncologists cooperating with the foundation, hence the diagnosis of these changes is really fast. However there was too little patients diagnosed with cancer to analyze whether the program had an impact on reducing the time to diagnosis in the pediatric population.

We identified in the study relatively high percentage of benign urinary tract pathologies which developing silently in asymptomatic children could have led to the more advanced stages of diseases leading to irreversible morphological and functional changes. Similarly high percentage of abnormalities in scrotal US supports the necessity of screening in boys up to 6 years, which thanks to early diagnosis and treatment can appear crucial for their fertility in adulthood. In these areas, we see the possibilities of using our research to develop specialized procedures to accelerate the treatment of these patients. On the other hand the high percentage of enlarged lymph nodes in this age group is a kind of physiology due to the activity of the immune system, vaccinations and frequent infections. No symptoms of lymph nodes 
malignancy and small percentage of other pathologies suggest that cervical US screening would probably not play a significant role in the population. The issue of costs is very important and reducing the amount of areas of interest could partially reduce these costs. Selection of patients to participate in the Program may constitute another limitation. The idea of the Program was to assess healthy children, but parents of children with pathologies recognized earlier could have had more motivation to participate in the studies.

\section{Conclusions}

US screening has proved to be very useful for the early identification cancerous as well as precancerous lesions, but due to the small percentage of the population covered by the screening, not all neoplasms were represented in sufficient numbers to draw definitive conclusions. Nevertheless, it is worth continuing this research even for a reason of effective early detection of other anomalies. However limiting the research to the abdominal cavity and testes in boys should be considered. The ideal would be the possibility of examining these children at least once a year, as non-invasive US examination can be used repeatedly.

\section{Funding}

- The mobile ambulance was shared by RMHC Chapter in Poland

\section{Conflict of interest}

- The authors declare they have no conflict of interest. 


\section{References}

1. Penta L, Cofini M, Lanciotti L, Leonardi A, Principi N, Esposito S. Hashimoto's Disease and Thyroid Cancer in Children: Are They Associated? Front. Endocrinol. 9:565. doi: 10.3389/fendo.2018.00565.

2. Qiao Y, Wang Y, Kang P, Li R, Liu Y, He W. The assessment of the accuracy of clinical preoperative lymph node. Medicine (2019) 98:4(e13778) doi:10.1097/MD.0000000000013778

3. Hong HS, Lee EH, Jeong SH, Park J, Lee H. Ultrasonography of Various Thyroid Diseases in Children and Adolescents: A Pictorial Essay. Korean J Radiol 2015;16(2):419-29

4. Ward E, De Santis C, Robbins A, Kohler B, Jemal A. Childhood and adolescent cancer statistics, 2014 Cancer J Clin 2014;64:83-103

5. Steliarova-Foucher E, Colombet M, Ries LA, Moreno F, Dolya A, Bray F et al. International incidence of childhood cancer, 2001-10: a population-based registry study. Lancet Oncol. 2017;18:719-31.

6. Kowalczyk JR, Dudkiewicz E, Balwierz W, Bogusławska-Jaworska J, Rokicka-Milewska R. Incidence of childhood cancers in Poland in 19951999. Med Sci Monit. 2002;8(8):587-90.

7. Gatta G, Botta L, Rossi S, Aareleid T, Bielska-Lasota M, Clavel J et al. Childhood cancer survival in europe 1999-2007: results of EUROCARE-5- a population-based study. Lancet Oncol. 2014;15(2):e52

8. Brasme JF, Morfouace M, Grill J,Martinot A, Amalberti R, Bons-Letouzey C et al. Delays in diagnosis of paediatric cancers: a systematic review and 
comparison with expert testimony in lawsuits. Lancet Oncol 2012; 13: e44559.

9. Steliarova-Foucher E, Fiedler MM, Colombet M, Lacour B, Kaatsch P, Pineros $\mathrm{M}$ et al. Changing geographical patterns and trends in cancer incidence in children and adolescents in Europe, 1991-2010 (Automated Childhood Cancer Information system): a poplation-based study Lancet Oncol. 2018;19(9):1159-69.

10. Autier P. Increasing incidence of cancer in children and competing risk. Lancet Oncol 2018;19(9):1136-37.

11. Zhanga J, Guoa F, Wanga L, Zhaoa W, Zhanga D, Yanga H et al. Screening and identification of non-inflammatory specific protein markers in Wilms'tumor tissues. Archives of Biochemistry and Biophysics 2019;676: $108-22$.

12. Tsuchiya M, Hayashida M, Yanagihara T, Yoshida J, Takeda S, Tatsuma N et al. Ultrasound screening for renal and urinary tract anomalies in healthy infants. Pediatr Int 2003;45:617-23.

13. Caiulo VA, Caiulo S, Gargasole C, Chiriaco G, Latini G, Cataldi L, Mele G. Ultrasound mass screening for congenital anomalies of the kidney and urinary tract. Pediatr Nephrol 2012;27:949-53.

14. Jedrzejewski G, Wozniak MM, Pawelec, A, Matera A, Kunach, M, Madej T et al. Ultrasound screening for neoplasms in children up to 6 years old. Medicine 2016;95(42),1-5.

15. Lewandowska A. Cancer epidemiology and prevention in children and adolescents - view thesis. Int J Pediatr Child Health 2013;1:19-26. 
16. Niedzielski JK, Oszukowska E, Słowikowska-Hilczer J: State of the art paper Undescended testis - current trends and guidelines: a review of the literature. Arch Med Sci 2016;12(3):667-677.

\section{Figure legends}

Fig 1. Ultrasound of the right kidney (left image) and left kidney (right image) in two year old patient. Bilateral kidney tumors with diameter under $30 \mathrm{~mm}$ in the right kidney and $40 \mathrm{~mm}$ in the left (callipers).

Fig 2. CT examination of the abdomen. (left figure). Both kidneys tumor (arrows). Preoperative CT study, after month of chemotherapy (right image).

Fig 3. Postoperative MRI study after 6 (left image) and 11 months of observation (right image). Postoperative scar in right kidney (arrow), no recurrence. 
Table 1. Abnormalities of the neck.

\begin{tabular}{|l|c|c|}
\hline Abnormalities of the neck & $\mathrm{N}$ & $\%$ \\
\hline All patients & 67169 & 8.30 \\
\hline Enlarged lymph nodes & 5572 & 1.40 \\
\hline Focal thyroid lesions & 944 & 0.29 \\
\hline Thyroid cyst & 196 & 0.35 \\
\hline Inhomogeneous thyroid echostructure & 240 & 0.05 \\
\hline Enlarged thyroid gland & 36 & 0.30 \\
\hline Hypoplasia of the thyroid gland & 202 & 0.04 \\
\hline Asymmetry of the thyroid lobes & 28 & 0.01 \\
\hline Agenesis of the thyroid lobe & 8 & 0.04 \\
\hline Neck hemangioma & 26 & 0.09 \\
\hline Cervical medial cyst & 60 & 0.03 \\
\hline Branchial cleft cyst & 20 & 0.09 \\
\hline Inhomogeneous salivary gland & 7419 & 11 \\
\hline Other & & \\
\hline All abnormalities & & \\
\hline
\end{tabular}


Table 2. Abdominal abnormalities.

\begin{tabular}{|c|c|c|}
\hline $\begin{array}{l}\text { Abdominal abnormalities } \\
\text { All patients }\end{array}$ & $\begin{array}{c}\mathrm{N} \\
67448\end{array}$ & $\%$ \\
\hline Hepatoblastoma & 1 & 0.0015 \\
\hline Hepatomegaly & 132 & 0.19 \\
\hline Abnormal liver echostructure (incl. fatty liver) & 34 & 0.05 \\
\hline Focal lesions of the liver & 20 & 0.03 \\
\hline Calcification in the liver & 8 & 0.01 \\
\hline Cyst of the liver & 26 & 0.04 \\
\hline Hemangioma of the liver & 22 & 0.03 \\
\hline Cholelithiasis & 52 & 0.08 \\
\hline Polyp of the gallbladder & 34 & 0.05 \\
\hline Splenomegaly & 662 & 0.98 \\
\hline Splenic cyst & 86 & 0.13 \\
\hline Focal lesion of the spleen & 32 & 0.05 \\
\hline Calcifications in the spleen & 12 & 0.02 \\
\hline Enlarged abdominal lymph nodes & 930 & 1.38 \\
\hline Abnormal pancreas echostructure & 12 & 0.02 \\
\hline Abdominal hernia & 114 & 0.17 \\
\hline Rib tumor & 1 & 0.0015 \\
\hline Other & 18 & 0.03 \\
\hline All abnormalities & 2195 & 3.25 \\
\hline
\end{tabular}


Table 3. Urinary tract abnormalities.

\begin{tabular}{|c|c|c|}
\hline $\begin{array}{l}\text { Urinary tract abnormalities } \\
\text { All patients }\end{array}$ & $\begin{array}{c}\mathrm{N} \\
67448\end{array}$ & $\%$ \\
\hline Agenesia of one kidney & 68 & 0.10 \\
\hline Hypoplasia of the kidney & 50 & 0.07 \\
\hline Dystopic kidney & 36 & 0.05 \\
\hline Hoerschoe kidney & 68 & 0.10 \\
\hline Duplication of the collecting systems & 426 & 0.63 \\
\hline Renal focal lesion & 14 & 0.02 \\
\hline Wilms tumor & 6 & 0.009 \\
\hline Renal cyst & 110 & 0.16 \\
\hline Dilation of the collecting system & 1074 & 1.60 \\
\hline Ureter dilation & 66 & 0.10 \\
\hline Asymmetry in kidney length & 92 & 0.14 \\
\hline Thickened bladder wall & 52 & 0.08 \\
\hline Ureterocele & 14 & 0.02 \\
\hline Urachus & 20 & 0.03 \\
\hline Renal stones & 40 & 0.06 \\
\hline Nefrolithiasis & 20 & 0.03 \\
\hline Hypertrophy of the kidney column & 162 & 0.24 \\
\hline Other & 14 & 0.02 \\
\hline All abnormalities & 2332 & 3.4 \\
\hline
\end{tabular}


Table 4. Reproductive system abnormalities

\begin{tabular}{|c|c|c|}
\hline Male reproductive system abnormalities & $\mathrm{N}$ & \\
\hline All patients & 34892 & $\%$ \\
\hline Undescended testis & 1434 & 4.1 \\
\hline Mobile testis & 3524 & 10.1 \\
\hline Agenesia of the testis & 56 & 0.16 \\
\hline Hypoplasia of the testis & 98 & 0.28 \\
\hline Abnormal testis echostructure & 20 & 0.05 \\
\hline Focal testis lesion & 16 & 0.05 \\
\hline Teratoma of the testis & 4 & 0.01 \\
\hline Microlithiasis & 358 & 1.03 \\
\hline Inguinal hernia & 82 & 0.24 \\
\hline Hydrocele testis & 592 & 1.7 \\
\hline Hydrocele of the spermatic cord & 118 & 0.34 \\
\hline Varicocele & 18 & 0.05 \\
\hline Cyst of the epididymis & 94 & 0.28 \\
\hline Calcifications in the epididymis & 60 & 0.16 \\
\hline All abnormalities & 6474 & 18.5 \\
\hline $\begin{array}{l}\text { Female reproductive system abnormalities } \\
\text { All patients }\end{array}$ & $\begin{array}{c}\mathrm{N} \\
18822 \\
\end{array}$ & $\%$ \\
\hline Ovarian cyst (>10mm) & 54 & 0.29 \\
\hline Enlarged follicles (3-10mm) & 38 & 0.20 \\
\hline Enlarged inguinal lymph nodes & 86 & 0.45 \\
\hline Fluid in the Douglas cavity & 68 & 0.36 \\
\hline All & 246 & 1.3 \\
\hline
\end{tabular}




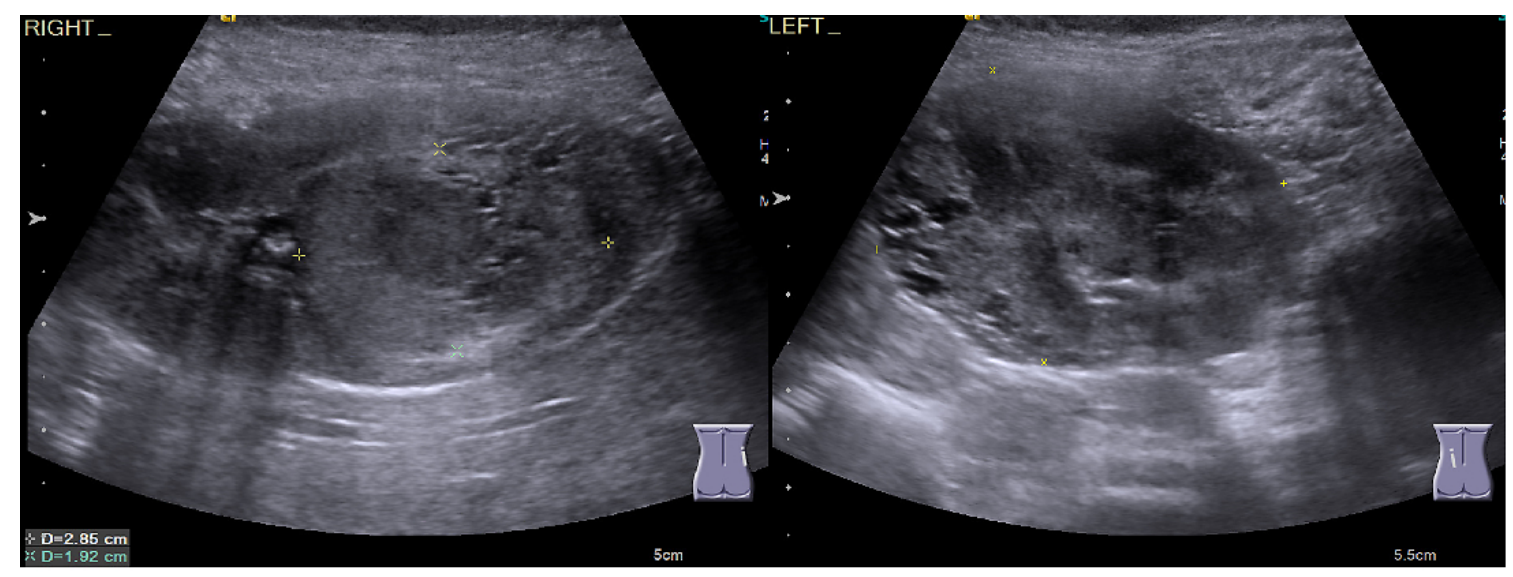

Ultrasound of the right kidney (left image) and left kidney (right image) in two year old patient. Bilateral kidney tumors with diameter under $30 \mathrm{~mm}$ in the right kidney and $40 \mathrm{~mm}$ in the left (callipers). 


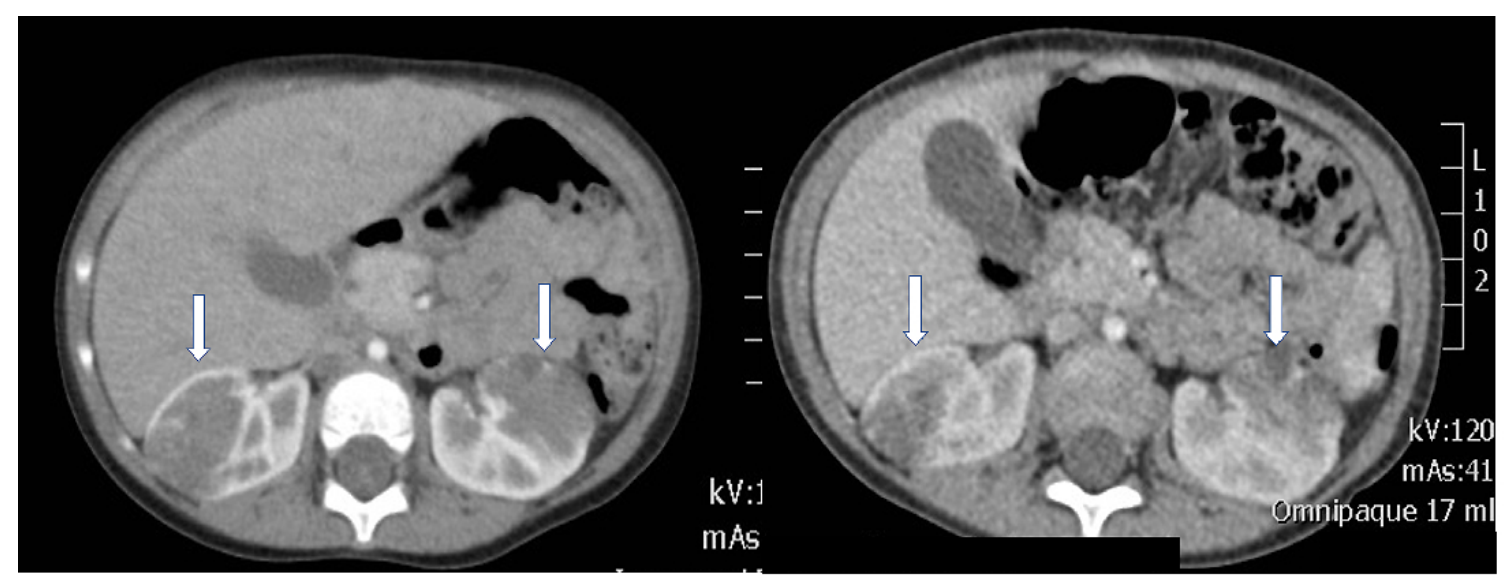

CT examination of the abdomen. (left figure). Both kidneys tumor (arrows). Preoperative CT study, after month of chemotherapy (right image). 


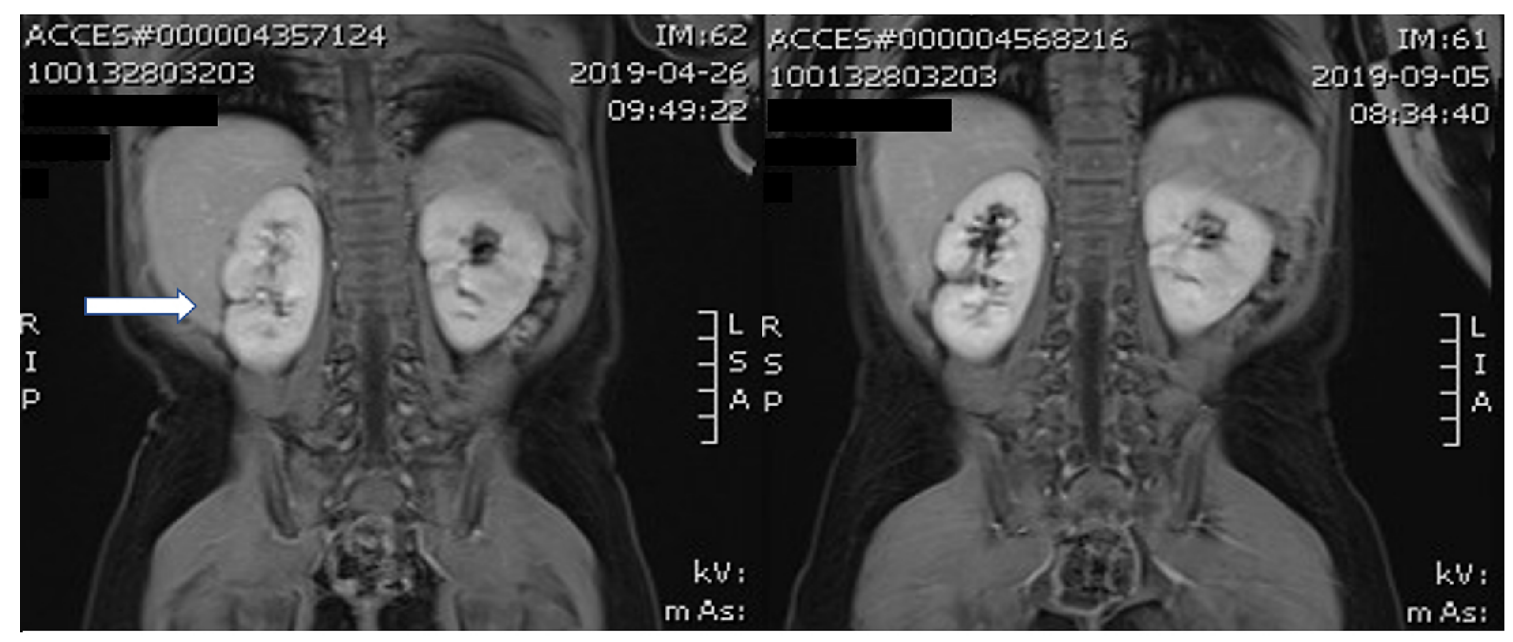

Postoperative MRI study after 6 (left image) and 11 months of observation (right image). Postoperative scar in right kidney (arrow), no recurrence. 